
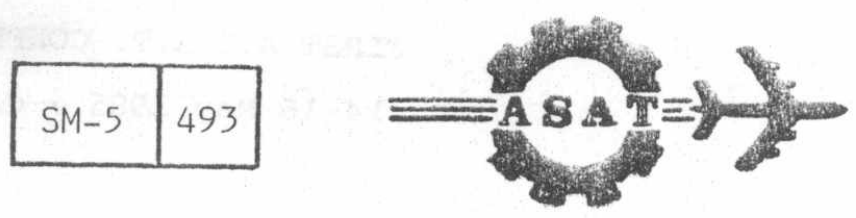

MILITARY TECHNICAL COLLEGE

CAIRO - EGYPT

EFFECT OF HEAT FLOW ACROSS THE WALLS OF POROUS CYLINDERS

ON THEIR STRENGTH AND OPTIMIZATION CRITERION

S.A.R.NAGA*

ABSTRACT

The use of porous materials, e.g. sintered metals, ceramics and refractories, has found its applications in many thermal problems. In which situations, the prediction of attained thermal stresses in porous media has to be carefully and accurately computed for proper and safe operation.

Assuming a steady heat flow across thick walled porous cylinder, the induced thermal stresses and consequently their influence on the total stress distribution due to existing pressures could be computed. By the aid of a specially devised computer program, the stress distribution in porous matrix of thick walled cylinder has been fully analysed and the selection of materials properties and cylinder dimensions have also been herein optimized.

\title{
INTRODUCTION
}

Cylinders with porous (permeable) walls have been previously studied [1] from the point of view of induced stresses due to both internal and external pressures. The presented resuIts confirmed that higher stress values could develop in porous cylinders compared to solid ones. Also, coating of a porous cylinder with impermeable coating or shrink fitting it in a solid cylinder would help higher stresses to exist in the porous matrix. Recently, the author has proposed an analytical solution to study the combined effect of both fluid pressure and temperature on the induced stresses in solid thick walled cylinders [2]. In this study, an optimum elastic and thermal properties of cylinder material has been attained as a function of the operating temperature and pressure with no dependence on the cylinder geometrical configu-
rations.

* Assistant Professor, Department of Materials Engineering, Faculty of Engineering, Zagazig University, Zagazig (Sharkia) Egypt. 
The effect of temperature on the behaviour of thick cylinders is two folds: deterioration in mechanical properties coupled with induced thermal stresses. The material composition of the cylinder may display weaker strength under high temperature situations [3]. However, for some porous nonmetallic materials e.g. ceramics, available data [4] showed a uniform strength up to temperature range of $1200^{\circ} \mathrm{C}$ beyond which a sharp decline in strength with temperature is fully recognised; a situation which was explained in terms of plastic deformation which may take place at very high temperatures. On the other hand, the temperature difference across a cylinder wall would impose a thermal stress pattern dependent on the thermal, physical and mechanical properties together with geometrical parameters [5]: a situation which may affect the design stresses under normal loading conditions.

Although porous materials can be found in various engineering applications imposed to heat flow, e.g.jet nozzles, filters, piping, furnaces, combustion chambers, boilers with internally coated with porous heat transfer surfaces, and other aerospace mechanical and chemical applications, little has been published to consider the case of porous cylinders subjected to both pressure difference and internal heat flow. Hence, it has been herein decided to investigate in full this case with due consideration to optimum material selection

\section{ANALYSIS AND OPTIMIZATION}

For porous permeable cylinders subjected to internal and external pressures, the normalized tangential stress is given in the form [1]:

$$
\begin{aligned}
\bar{\sigma}_{\theta p}=-1+\left(1-\frac{p_{0}}{p_{i}}\right)\left[\frac{1}{3(1-\nu)\left(1-R^{2}\right)\{}\right. & (5-4 \nu)\left(1+\frac{r_{i}^{2}}{r^{2}}\right) \\
& +(2-\gamma)(1+R) R \\
& \left.\left.-(1+\gamma)(1+R) R\left(\frac{r}{r_{i}}\right)\right\}\right] 1
\end{aligned}
$$

Assuming a steady heat flow and isothermal condition through the porous cylinder wall, the temperature gradient can be described as such,

$\mathrm{T}=\frac{\mathrm{T}_{\mathrm{i}}-\mathrm{T}_{\mathrm{O}}}{\ln \left(\frac{1}{\mathrm{R}}\right)} \ln \left(\frac{\mathrm{r}_{\mathrm{O}}}{\mathrm{r}}\right)$

Adopting this equation and applying it to the stress equations resulting from heat flow across thick cylinders $! 5 !$, the normalized tangential thermal stress can be presented by:

$$
\bar{\sigma}_{\theta t}=-\frac{1}{2(1-\nu) \ln (R)}\left\{1-\ln \left(\frac{r_{0}}{r}\right)+\frac{R^{2} \ln (R)}{\left(1-R^{2}\right)}\left(1+\frac{r_{0}^{2}}{r^{2}}\right)\right\}
$$

The combined effect of both the pressure and temperature on the tangential stress would result by superimposing equation 1 
onto equation 3 to give the total tangential stress distribution $\vec{\sigma}_{\Theta T}$

$\bar{\sigma}_{\theta T}=\frac{\sigma_{\theta T}}{p_{i}}=\bar{\sigma}_{\theta p}+\bar{\sigma}_{\theta t} \propto \mathrm{E}\left(\frac{\mathrm{T}_{i}-\mathrm{T}_{0}}{p_{i}}\right)$

From equations 1,3 , and 4 the resultant tangential stress distribution can readily be computed.

An optimization can, thus, be achieved with the objective of minimizing the resultant maximum tangential stress values and in the mean time controlling the stress distribution to give the best uniform shape. This objective has been herein performed by directly equating the resultant stress, $\sigma_{G \mathrm{~T}}$ at inner and outer cylinder surfaces. Which procedure renders an optimization criterion in the form:

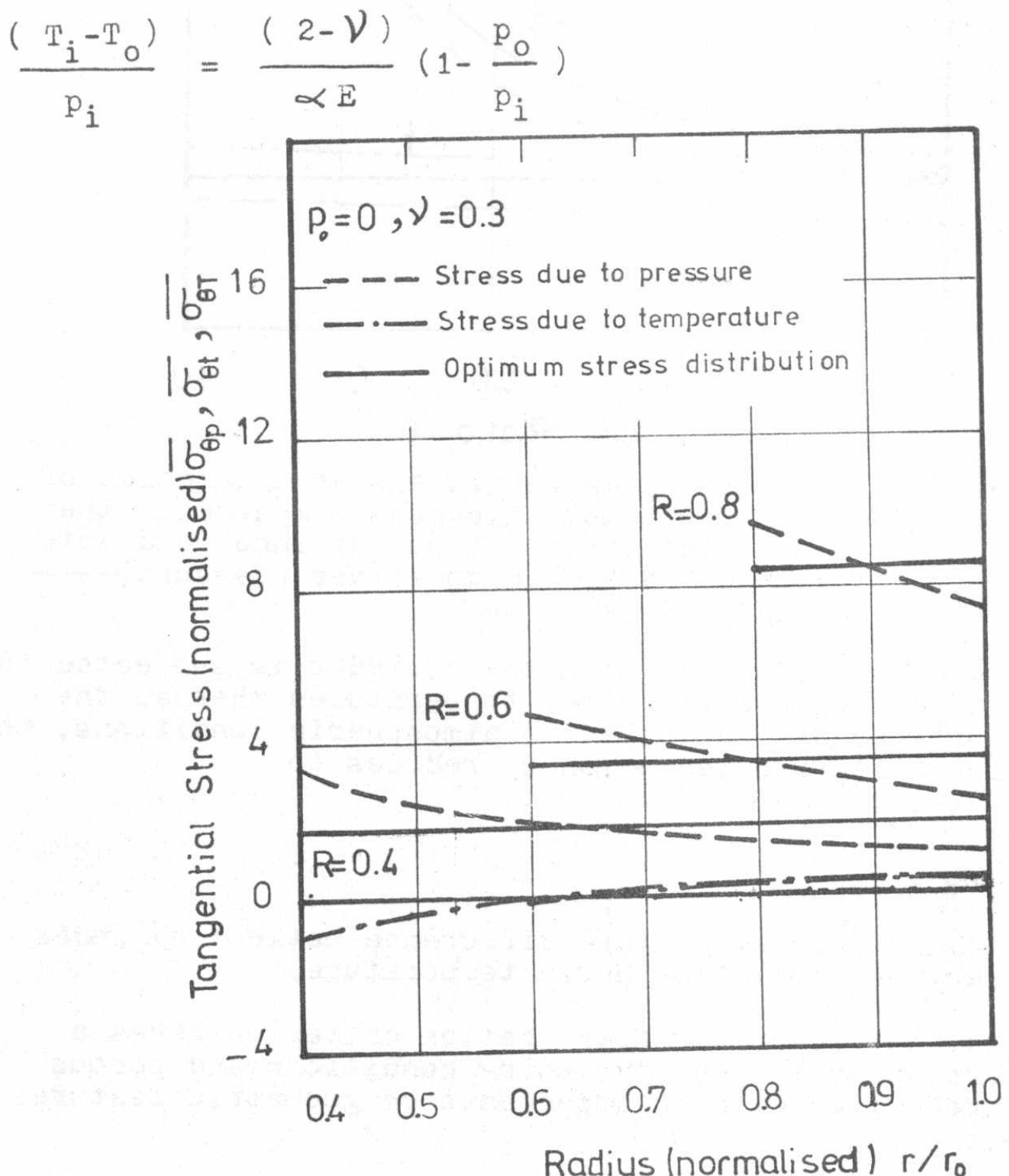

Figure (1); Effect of internal pressure and temperature on tangential stress distribution 


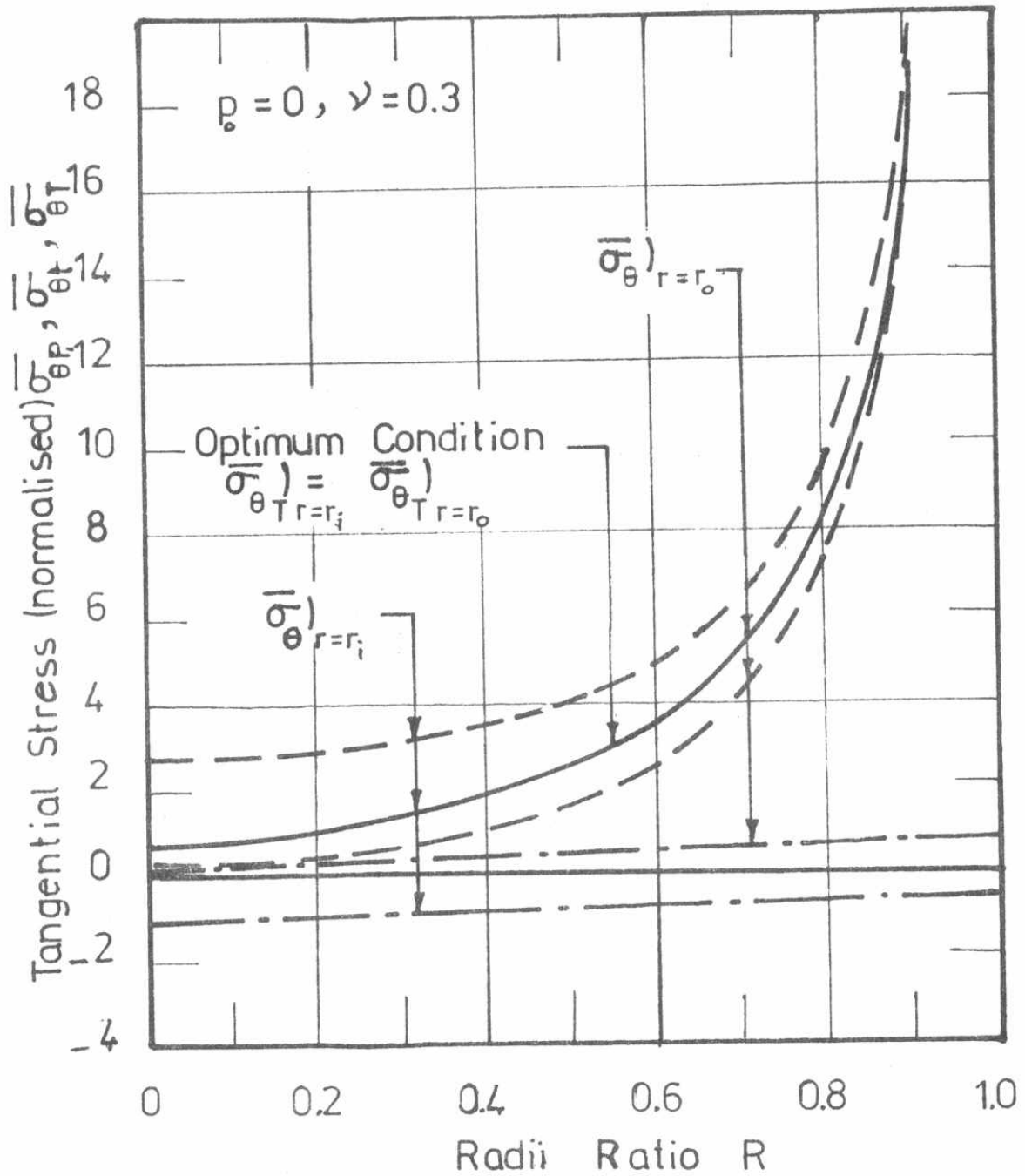

Figure (2): uptimum tangential stress as a function of porous cylinder thickness compared to the tangential stress values at inner and outer cylinder radii due to ejther pressure(- - ) or temperature $(-\cdots)$

In the more common case in which the cylinder is subjected to internal fluid pressure at raised temperatures whereas the external surfaces are subjected to atmospheric conditions, the optimization criterion, equation 5, reduces to:

$\left(\frac{\mathrm{T}_{i}}{\mathrm{p}_{i}}\right)=\frac{(2-\nu)}{\alpha E}$

where $\mathrm{T}_{i}$ stands for temperature difference between cylinder inner temperature and atmospheric temperature,

This simple, but powerful, optimization criterion gives a direct correlation between operating conditions and porous material properties with no dependence on geometric features of the cylinder.

\section{RESULTS AND DISCUSSION}

Results attained to describe the combined effect of internal pressure and heat on the tangential stress distribution 


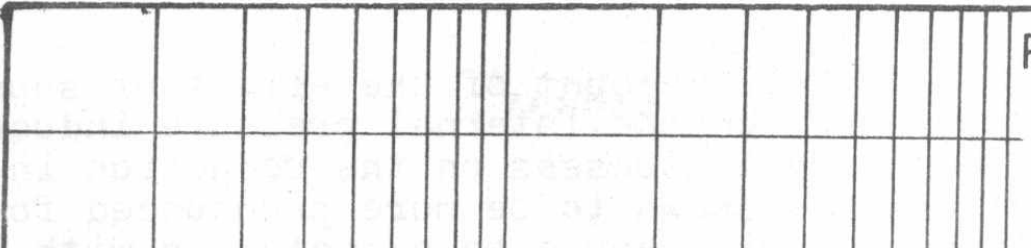

$R=0.9$

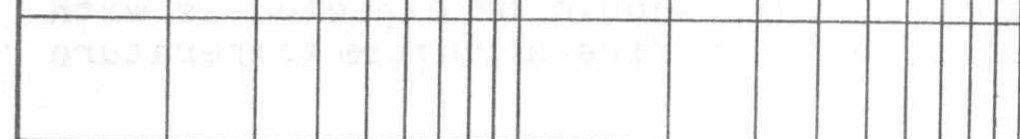

0.5
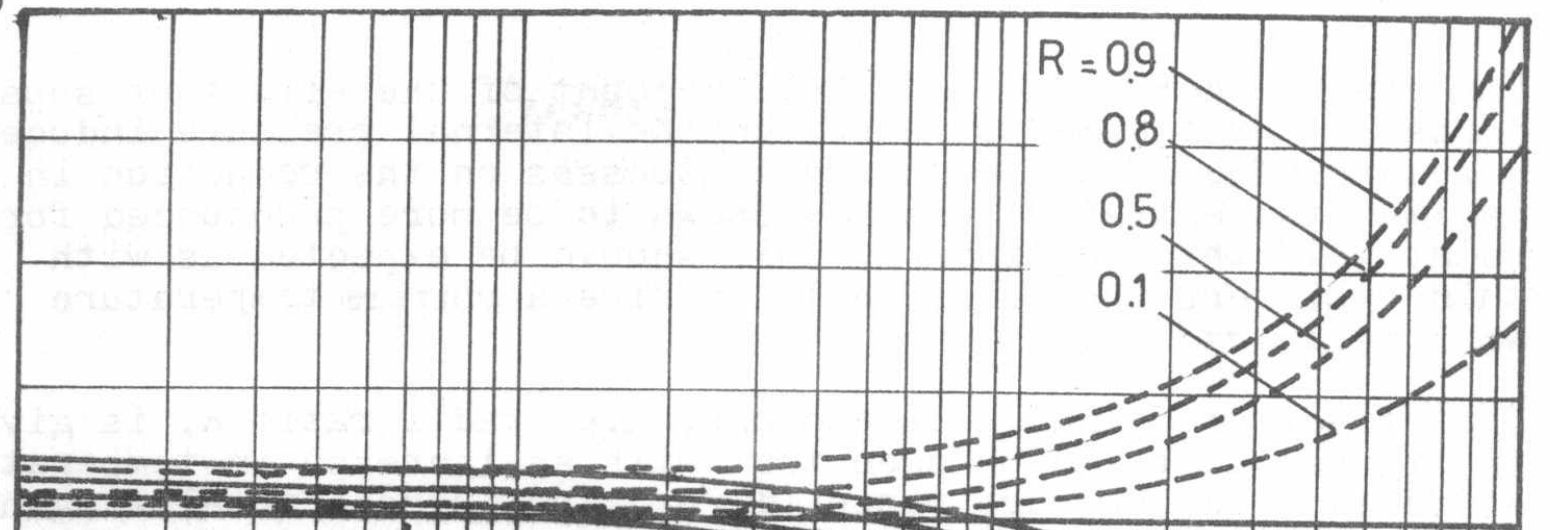

0.1
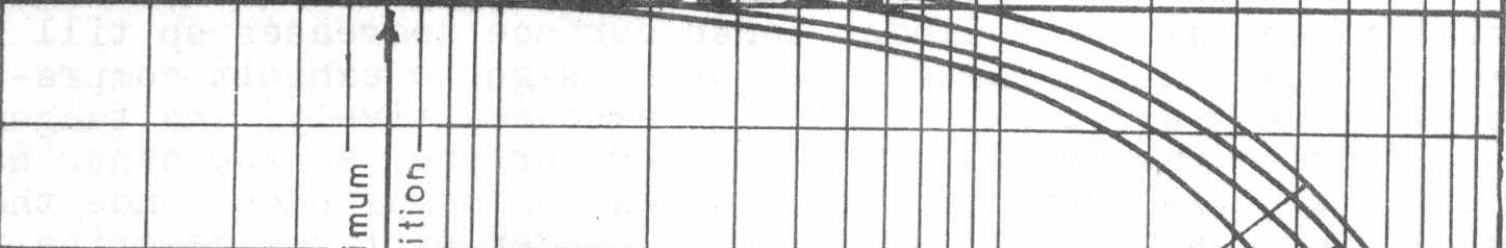

\begin{tabular}{l}
\hline \\
\hline
\end{tabular}

点
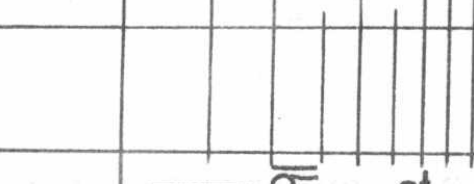

1

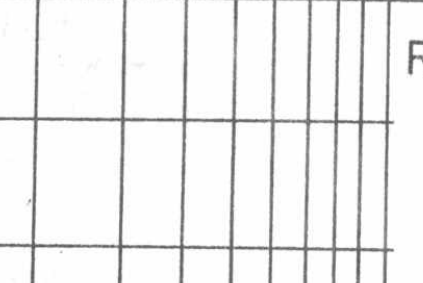

$R=0.9$

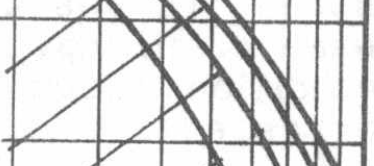

0.8

0.5

0.1 $--\frac{\theta}{\sigma_{\theta T}}$ at $r=r_{0}$<smiles>O=[W]O</smiles>

Temperature-Pressure Ratio $\left(T_{i}-T_{0}\right) / P_{i}$

Figure (3): Graphical assessment of optimum condition

in a porous cylinder are displayed in Figure 1. The superposition of thermal stresses on the induced tangential stress due to internal pressure has shown to produce a more likely uniform stress distribution across the cylinder wall if the proposed optimimum criterion is adopted. This uniformity in the stress distribution, as shown in Figure 1, permits a maxinum utilization of the material. Any departure from the proposed optimum condition would lead to a higher developed stresses either at the inner cylinder or the outer cylinder surfaces. This ensures that the novel optimum objective herein assumed inwhich the combined total stress values at cylinder inner and outer surfaces have to be identical, renders the minimum and almost uniform tangential stress distribution. In practice, however, the optimum design criterion can be put into effect by controlling the temperature difference across the cylinder wall to justify a temperature to pressure ratio analogous to that governing the optimum criterion, equation 5 . However, another possibility is to optimize the selection of porous material properties sothat the elastic and thermal properties satisfy the design criterion. 
The graphs in Figure2 show full account of the effect of superimposing the thermal stresses on the internal pressure induced stresses. The effect of thermal stresses on the reduction in maximum tangential stress has shown to be more pronounced for relatively thick cylinders. This should be expected as with thin cylinders the walls would decribe a uniform temperature with zero gradient.

The effect of cylinder dimensions, i.e. radii ratio $k$, is given in figure 3. It can be seen that with an increase in temperature difference to pressure ratio $\left(\mathrm{T}_{i} / \mathrm{p}_{i}\right)$, the induced total tangential stress at the cylinder inner surface decreases up till zero value and the reverts to change sign to exhibit compressive stress and continues to increase negatively. The tangential stress values at outer cylinder surface, on the other hand, retain tensile behaviour with increasing magnitudes. Dnce the stress values at inner and outer cylinder surfaces describe the same magnitude, the optimum condition prevails. The optimum condition occurs at a definite temperature to pressure ratio irrespective of cylinder geometry.

\section{CONCLUSIONS}

Both the fluid pressure and temperature gradient across the walls of porous cylinder would affect the strength and the induced stress distribution. Optimum criterion independent of the cylinder geometry could be derived to correlate working conditions th material properties in an endeavour to maximize material utilization by assuming a minimum and uniform developed stress distribution.

\section{REFERENCES}

1. Naga,S.A.R.,"Analytical Study of Thick Walled Forous Cylinder" Trans. ASME, J. of Engineering Materials and Technology, Vol.107.90-94, (1985)

2. Naga,S.A.R.,"Stress Analysis and uptimization of Thick walled Cylinders Subjected to Steady Heat Fluw", Faper presented at the 2nd Conf. on Theoretical and Applied Wechanics, Cairo Narch 11-13, 1985, Academy of Scientific Research and Tech.

3. Properties and Selection of Metals, ASM Netals Handbook, Vol.1, American Society for Vietals, 8th Ed.,(1961)

4. Ryshkewitch,E., "Oxide Ceramics", Academic Fress,NewYork, (1960)

5. Fung,Y.C.,"Foundations of Solid Mechanics", Frintice-hall Inc, New Delhi, (1968)

\section{NOMENCLATURE}

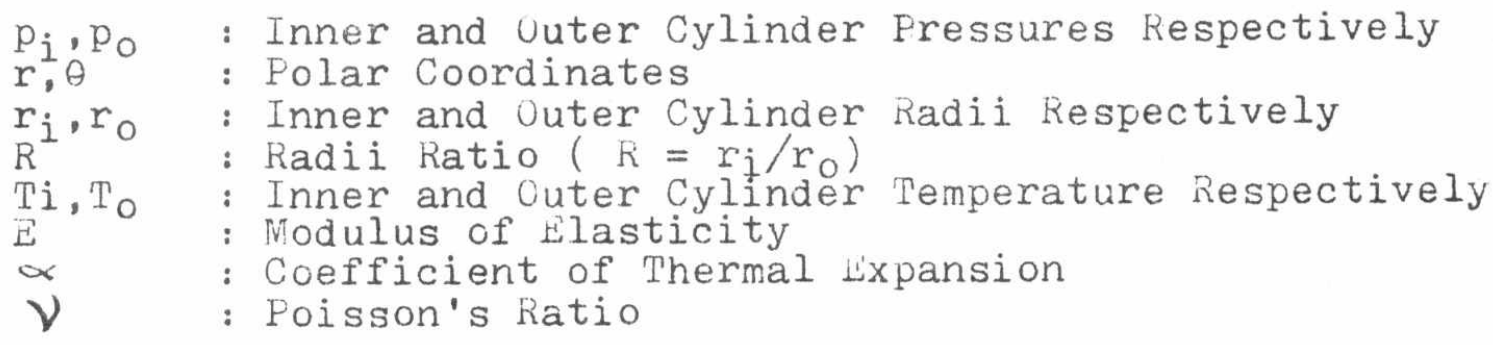


$\sigma_{\theta} \quad$ : Tangential Stress

$\sigma \frac{\theta}{\theta p} \quad$ : Tangential Stress due to pressure

$\overline{\sigma_{\theta p}}$

: Normalized Tangential Stress Due to pressure $\left(\bar{\sigma}_{\theta p}=\frac{\sigma_{\theta} p}{p_{i}}\right)$

$\sigma_{\theta} t$

: Tangential Stress due to temperature difference

$\overline{\sigma_{t}}$

: Normalized Tangential Stress due to temperature difference $\left(\bar{\sigma}_{t}=\sigma_{\theta t} / \alpha E T_{i}\right)$

$\sigma_{\theta T}$ : Total Tangential Stress

$\overline{\sigma_{\theta T}}$

: Normalized Total Tangential Stress $\left(\overrightarrow{\sigma_{\theta T}}=\sigma_{\theta T} / p_{i}\right)$ 